\title{
Henry VI, première partie, la guerre de Cent ans, Jeanne D'Arc
}

\section{Philippe Contamine}

\section{(2) OpenEdition}

\section{Journals}

\section{Édition électronique}

URL : http://journals.openedition.org/shakespeare/1217

DOI : 10.4000/shakespeare. 1217

ISSN : 2271-6424

\section{Éditeur}

Société Française Shakespeare

\section{Édition imprimée}

Date de publication : 1 novembre 1990

Pagination : 113-127

Référence électronique

Philippe Contamine, "Henry VI, première partie, la guerre de Cent ans, Jeanne D'Arc », Actes des congrès de la Société française Shakespeare [En ligne], 8| 1990, mis en ligne le 01 janvier 2007, consulté le 01 mai 2019. URL : http://journals.openedition.org/shakespeare/1217 ; DOI : 10.4000/ shakespeare.1217 


\section{SHAKESPEARE \\ ET LA GUERRE}

Ouvrage publié avec le concours de la Commission

des Communautés Européennes 
Illustration de la couverture : DERRICKE'S Image of Ireland, 1586 


\section{Henry VI, première partie, la guerre de Cent ans, Jeanne d'Arc}

À l'initiative de Madame Jones-Davies, votre colloque a fait appel à un historien, et, qui pis est, à un médiéviste, pour qu'il vous apporte son point de vue sur la façon dont Shakespeare a évoqué le long conflit qui opposa, entre le milieu du XIV ${ }^{\mathrm{e}}$ et le milieu du XV siècle, les rois de France aux rois d'Angleterre, les Valois aux Plantagenêts puis aux Lancastres, le royaume de France au royaume d'Angleterre, les Français aux Anglais conflit qu'on a pris l'habitude, depuis le XIX ${ }^{e}$ siècle, d'appeler la guerre de Cent ans.

Ce faisant, vous avez été bien imprudents. car, à première vue, comment un historien peut-il traiter semblable sujet sinon en s'interrogeant, professionnellement, sur les procédés utilisés par Shakespeare pour évoquer le dit conflit, le mettre en scène, et sur sa fidélité plus ou moins grande à l'histoire? Autrement dit, le mode d'approche adopté par Shakespeare pour présenter cette série d'événements est-il conforme à la "vérité historique»? Par «vérité historique», l'on entendra ici non seulement l'analyse que font de la guerre de Cent ans les historiens actuels, dans ses causes, dans ses objectifs, dans son déroulement, dans son caractère ${ }^{1}$, mais aussi l'enseignement et les renseignements fournis par les deux grandes sources dont nous savons qu'elles ont servi en l'occurrence de base documentaire au dramaturge: Edward Hall, The Union of the two noble and illustre families of Lancastre and York (1548-1550: réimprimé en 1809) et Raphael Holinshed, The Chronicles of Englande, Scotlande and Irelande (2e éd. 1587). Et l'on voit d'emblée 
ce que peut avoir de naïf, d'insolent, sinon d'insolite, une démarche de ce genre qui fait tout simplement fi de la souveraine liberté du poète. C'est dire que cette démarche sera entreprise sans vergogne mais dans la pleine conscience de ses limites.

Encore qu'aucune donnée chronologique précise ne soit fournie, la pièce couvre la période 1422-1444. Elle débute par les obsèques de Henry $V$, dans le cadre de l'abbaye de Westminster, et s'achève par l'annonce d'une paix avec la France, avec le «dauphin» Charles, et du mariage de Henry VI, fils de Henry V, avec Marguerite d'Anjou, fille du roi René de Naples (ou de Sicile). Il n'empêche que sont longuement, dramatiquement évoqués le dernier combat et la mort de John Talbot, comte de Shrewsbury, alors que, dans la réalité, ces événements se sont situés en 1453, lors de la bataille de Castillon. Première question, laissée pour le moment sans réponse: pourquoi cette entorse, patente et sérieuse, à la chronologie?

À l'intérieur de ces quelque vingt années, les scènes, nombreuses, se déroulent de part et d'autre de la Manche: à Londres (Westminster, ainsi qu'on vient de le voir, devant et dans la Tour, dans le jardin du Temple, avec le superbe épisode des deux roses, dans le palais royal, dans la salle du Parlement), à Paris (dans le palais et dans la grande salle), mais aussi devant et dans Orléans, devant Rouen, Bordeaux et Angers, dans les plaines de Gascogne et dans celles d'Anjou, dans le camp anglais (Anjou et Gascogne), enfin dans un château appartenant à la comtesse d'Auvergne. Autant de lieux de pouvoir et d'histoire: pour le thème qui nous retient ici - la guerre - , il est intéressant de voir apparaître, presque à égalité, les sièges de ville et les champs de bataille, c'est-à-dire, sans surprise, les cadres essentiels du déroulement de la guerre de Cent ans. Il y a des agonies, des cadavres, des obsèques, des rixes et des combats singuliers, des batailles rangées, des assauts et des retraites, des coups de canon, tout un contentieux entre les Français et les Anglais mais surtout entre les protagonistes anglais eux-mêmes, car, 
autant que l'évocation de la guerre entre les Valois et les Lancastres, la pièce propose une préfiguration des guerres des Roses ${ }^{2}$.

La pièce commence dans l'affliction et la crainte: Henry V n'est plus, rien ne va plus. Les nouvelles de France sont apportées à Westminster à l'intention des nouveaux maîtres du royaume d'Angleterre - puisque l'enfant Henry ne peut effectivement régner - : l'oncle du roi défunt, Henry Beaufort, un prélat ambitieux et habile que l'on verra plus tard devenir cardinal tout en conservant son évêché de Winchester, Humphrey, duc de Gloucester, un va-t-en guerre sans scrupule dans l'exercice de son office de protecteur du royaume, Thomas Beaufort, duc d'Exeter, gouverneur de Henry VI, Jean, le sage, duc de Bedford, régent du royaume de France, qui va, par la suite, mourir sous nos yeux de vieillesse, plus quelques autres grands seigneurs destinés à figurer ultérieurement dans différents épisodes de la pièce. Tout cela historiquement bien vu: les forces en présence, les options politiques et militaires sont fermement dessinées, et, à quelques nuances près, la critique historique ne peut ici qu'approuver.

La situation est donc plus qu'inquiétante, presque désastreuse: non seulement la plupart des grandes villes du royaume de France sont perdues - Orléans, Paris, Poitiers, Reims, Rouen -, non seulement presque toute la France s'est révoltée contre la paix anglaise, non seulement le dauphin Charles s'est fait couronner à Reims, mais Lord Talbot, ce parangon de chevalerie, ce chef de guerre aussi preux qu'efficace, ce champion incontesté de la cause anglaise, ce fléau des Français, vient d'être battu et fait prisonnier - l'on apprendra plus tard que c'est à la bataille de Patay - à cause de la lâcheté de Sir John Fastolf, indigne chevalier de la Jarretière. Il est grand temps de réagir. Le comte de Salisbury, qui assiège Orléans, crie à l'aide. Voilà qui décide Bedford de traverser la Manche à la tête d'une armée de secours.

La pièce finit, en revanche, de façon relativement optimiste: certes le grand Talbot est mort, mais les Fran- 
çais - ruse ou lassitude? - acceptent la paix : bien que reconnu dans la moitié de son royaume, le dauphin Charles consent à faire acte d'allégeance au roi d'Angleterre: il ne sera plus chez lui que vice-roi. Signe de la réconciliation: rompant un avantageux projet d'alliance matrimoniale avec la fille du comte d'Armagnac, Henry VI est tout content d'épouser la belle Marguerite d'Anjou. Il est vrai que déjà les nuages s'amoncèlent: la couronne d'Angleterre n'a pas fait là une bonne affaire car Marguerite n'a pas de dot. Pis encore : elle était la prisonnière de guerre de Suffolk, mais le sot l'a libérée afin d'obtenir son consentement au mariage avec Henry VI, et surtout se dessine le caractère de ce dernier: naïf, dévot, manquant d'autorité, avec une allergie viscérale pour toute forme de conflit.

Naturellement, au cours de la pièce, une foule de personnages apparaissent - et disparaissent - , mais deux d'entre eux dominent : le couple fondamental Talbot et la Pucelle.

Mention est faite de Talbot dès la première scène de l'acte I, à propos du combat inégal entre «the stout Lord Talbot», le vaillant Talbot, qui, tel un diable, précipitait par centaines les Français aux enfers mais qui, à la fin, submergé par le nombre, fut vaincu et fait prisonnier, un vil Wallon, profitant de la débâcle générale, l'ayant lâchement frappé d'une lance par derrière. On le retrouve ensuite, cette fois en personne, alors qu'il inspecte les fortifications d'Orléans, en compagnie de Salisbury. Luimême raconte comment il a été libéré, contre Poton de Saintrailles. Au début de sa captivité, les Français ont eu le front de proposer un échange avec un simple homme du rang, mais c'était insulter à son honneur, mettre en doute le prix auquel lui-même s'estime. Il a refusé dignement. Car même chez l'adversaire sa réputation est énorme. On le redoute comme un véritable épouvantail. Aussi, pour ruiner par la dérision la terreur sacrée qu'il inspire, les Français l'ont-ils offert au public en pleine place du marché, comme un voleur cloué au pilori. Mais son attitude, ses gestes menaçants ont suffi à tenir en respect ses 
gardiens, qui dès lors ont redoublé de précautions. Le voilà enfin libre, prêt à reprendre le combat. C'est alors qu'un coup de canon, tiré, presque sans le vouloir, par un jeune garçon d'Orléans, frappe à ses côtés Salisbury, lequel s'écroule, blessé mortellement. Talbot, sur le cadavre de son camarade, jure de le venger et, tel un nouveau Néron, de contempler un jour Orléans en feu. Rien ne l'arrête. Il affronte la sorcière Jeanne d'Arc en un combat singulier, qui se termine par un match nul, mais réussit à reprendre Orléans, décidant alors, comme promis, d'ériger dans la cathédrale un tombeau pour Salisbury sur lequel sera inscrit le récit du sac de la ville.

Suit une scène assez curieuse. La comtesse d'Auvergne, attentive depuis longtemps à sa réputation, l'invite à lui rendre visite dans son château. Talbot accepte, sans être dupe. Lors de leur rencontre, la comtesse, qui ne le connaît que par son portrait, affecte d'être déçue par sa piètre apparence physique: elle s'attendait à un autre Hercule, à un nouvel Hector, et la voilà en présence d'un enfant, d'un avorton, presque d'un nain:

Alas, this is a child, a silly dwarf!

It cannot be this weak and writhled shrimp

Should strike such terror to his enemies (II.iii).

S'étant vu déclaré qu'il était son prisonnier, Talbot éclate de rire: la comtesse ne tient et ne retient que son ombre, car sa substance, son cadre tout entier, sa force, ses bras, ses muscles, ce sont ses vaillants soldats, lesquels, au même moment, accourent en masse, au son des tambours, pour le délivrer. Certes, Talbot serait en droit de se venger d'un tour aussi misérable: mais, en généreux chevalier, il se contentera de boire le vin de la comtesse, car , n'est-ce pas?, un soldat est toujours assoiffé... Outre une allusion possible au physique de l'acteur auquel Shakespeare peut confier le rôle de Talbot, sans doute faut-il voir là un apologue destiné à montrer que seule «l'union fait la force». 
Encore par ruse, la Pucelle est parvenue à s'emparer de Rouen. Pour lutter contre elle Talbot est le plus ardent. Sa colère explose: «Pucelle, that witch, that damned sorceress», «vile fiend and shameless courtezan», «foul fiend of France and hag of all despite». Même la Pucelle est impressionnée: «If Talbot do but thunder, rain will follow». De fait, la ville est reprise, et le duc de Bourgogne renoue solennellement son alliance avec les Anglais.

Talbot est le contraire d'un courtisan: depuis des années qu'il se bat, rudement, en France, il n'a pas eu l'occasion de retourner en Angleterre et d'y voir le roi. C'est donc seulement lors du sacre de Henry VI à Paris que se place leur première rencontre. Le voilà enfin, ce héros qui a réduit pour son maître cinquante forteresses, douze cités, sept puissantes villes fortifiées, qui lui a livré cinq cent prisonniers de marque. aussi est-il fait sur-lechamp comte de Shrewsbury. Mais Fastolf survient: en présence du roi ébahi et silencieux, Talbot, lui reprochant véhémentement sa conduite à Patay, lui arrache la Jarretière, il le dégrade de la chevalerie.

Talbot est maintenant sous Bordeaux. Tel un cerf fou de rage, il est encerclé par la meute des Français. Il sent qu'il est trop tard. Son énergie est celle du désespoir. Encore une dernière fois, pour Dieu, pour saint Georges, pour le droit, pour les couleurs anglaises! Dans ces circonstances, a lieu la rencontre entre les deux Talbot, le père et le fils, qui font assaut de générosité et de courage. Leur attitude est à la fois celle de chevaliers chrétiens et de héros de l'Antiquité. En vain le père s'efforce-t-il de renvoyer le fils pendant qu'il en est encore temps, en vain le fils cherche-t-il à préserver le père. Tous deux meurent, solidaires d'un même destin lucidement affronté. Il ne reste plus qu'à l'un de leurs compagnons d'armes, Sir William Lucy, d'annoncer pompeusement la mort de Talbot au dauphin Charles en personne:

But where's the great Alcide of the field, Valiant Lord Talbot, Earl of Shrewsbury, 
Created Earl of Washford, Waterford and

Lord Talbot of Goodrig and Urchinfield, Valence; Lord Strange of Blackmere, Lord Verdun of Alton, Lord Cromwell of Wingfield, Lord Furnivall of Sheffield, The thrice-victorious Lord of Falconbridge; Knight of the noble order of Saint-George Worthy Saint Michael and the Golden Fleece; Great marshal to Henry the Sixth Of all his wars within the realm of France? (IV.vii).

Notons que ce soldat exemplaire, ce chef incomparable, ce modèle de toute chevalerie, ne se mêle d'aucune manière des querelles qui opposent, autour du jeune et faible roi, les seigneurs d'Angleterre. Dédaignant la rose rouge comme la rose blanche, il est au-dessus des factions. Son seul parti, c'est l'Angleterre.

L'anti-Talbot dans la pièce de Shakespeare, c'est «la Pucelle». L'épisode avec la comtesse d'Auvergne esquisse, d'un sens, un contre-portrait de Talbot, personnage de petite apparence dont la réputation serait surfaite. Mais il ne s'agit que d'une esquisse. Pour Jeanne d'Arc au contraire, deux images se trouvent pleinement développées : telle qu'elle apparaît à ses partisans, et telle qu'elle apparaît à ses adversaires. Mais la vision de ses adversaires est la plus crédible, présentée de telle sorte que, pour le public, c'est elle seule qui corresponde à la vérité.

La scène se passe sous les murs d'Orléans, entre la dauphin Charles et les ducs d'Alençon et d'Anjou, à la tête d'une armée de secours destinée à délivrer la ville qu'assiège Salisbury. Les Anglais ne sont plus que d'inconsistants fantômes. Ils se bornent à attaquer la ville une heure par mois. Sans doute leur manquent le porridge et leurs gros beefsteaks. Quant à leur chef, Salisbury, il est fou. Mais les Français vont aussitôt faire la preuve de leur légèreté, de leur manque de ténacité - l'un des thèmes de la pièce. On apprend en effet qu'une bataille a eu lieu et 
que ces Anglais faméliques et anémiques, soudain réveillés, se sont battus comme des lions, des Samson, des Goliath, des Olivier, des Roland. Et d'ailleurs Froissart les montre déjà ainsi, sous le règne d'Édouard III. Ils ne se sont pas laissés décourager par leur infériorité numérique: autre thème récurrent. Ils ont frappé comme des robots ou plutôt, selon la comparaison employée, comme des horloges mécaniques. Du coup, le dauphin décide de quitter le siège. Alors survient le bâtard d'Orléans: ne décampez pas, j'amène avec moi une sainte fille («Holy maid $\gg$ ), à laquelle certaine vision du Ciel a enjoint de faire lever le siège d'Orléans et de chasser de France les Anglais. Elle est animée d'un esprit de prophétie qui surpasse celui des neuf Sibylles de Rome. Elle décrit le passé et l'avenir. Cela dit, comment le Bâtard a-t-il découvert cette prophétesse, nous ne le savons pas. Rien dans la pièce ne suggère que la Pucelle ait été suscitée - manipulée - par exemple par le Bâtard lui-même afin de redonner courage aux Français grâce à cet artifice. Or, telle était, plus ou moins, la thèse «bourguignonne», formulée dès le $X^{\mathrm{e}}$ siècle, selon laquelle Robert de Baudricourt avait été à l'origine de cette supercherie ou de cette mise en scène $e^{3}$.

Jeanne la Pucelle fait alors son entrée. Elle déjoue la ruse qui consistait à lui faire prendre René d'Anjou pour le dauphin, et, sous l'œil médusé des courtisans, demeurés à distance respectueuse, elle a un long entretien en tête-àtête avec Charles. Elle est la fille d'un berger, et c'est alors qu'elle gardait les moutons de son père que la Mère de Dieu lui est apparue, l'enjoignant de quitter son vil état pour libérer son pays du malheur : «To leave my base vocation / And free my country from calamity». En même temps, elle qui avait le visage noirci par le soleil est transfigurée: elle devient blonde, blanche et belle. Ainsi, dans toute la pièce, Jeanne d'Arc apparaît comme une jolie et forte fille, ce qui contraste avec Talbot, plutôt disgracié. Or, selon l'une des sources de Shakespeare, la chronique de Hall, si elle avait gardé sa virginité, c'était soit par vœu soit parce que personne n'en voulait, en raison de sa 
laideur («because of her foule face»). Lidée de Shakespeare est bien de mettre en scène une Jeanne d'Arc dont l'ascendant sur son entourage s'explique d'abord par l'attrait, et plus précisément, l'attrait sexuel qu'elle exerce.

Pour lui accorder sa confiance, le dauphin ne lui demande pas de signe, il ne cherche pas à être rassuré sur la légitimité de sa naissance ou sur ses droits à la couronne: la preuve de sa mission, ce sera un combat singulier qu'il livrera avec elle et dont elle sortira victorieuse. Du coup le dauphin tombe amoureux d'elle: il la compare non seulement à une Déborah, à une Amazone, mais à Vénus.

Après la victoire remportée sous Orléans, Charles s'enthousiasme: «France, triumph in thy glorious prophetess». Il invite tous les prêtres, tous les religieux du royaume à se rendre en procession pour chanter ses louanges. Quand elle sera morte, il édifiera pour elle une pyramide en guise de tombeau.

Her ashes in an urn more precious

Than the rich-jewel'd coffer of Darius,

Transported shall be at high festivals

Before the kings and queens of France.

No longer on Saint Denis will we cry,

But Joan la Pucelle shall be France's saint (I.vi).

Louanges étonnantes, propos sublimes qui semblent préfigurer le culte à la fois religieux et patriotique que la majorité de l'opinion française va rendre à Jeanne, avec de plus en plus de ferveur, à partir de 1870 et durant le demisiècle qui suivit. Dès lors n'est-on pas amené à penser que Shakespeare a ici célébré la Pucelle dans des termes qu'on ne saurait retrouver à semblable époque même chez ses plus ardents thuriféraires de l'autre côté de la Manche ${ }^{4}$ ? Sans doute. Mais une autre lecture de ce passage est possible. Pourquoi Shakespeare, par les propos qu'il place dans la bouche du dauphin, n'aurait-il pas voulu surtout montrer l'idolâtrie des Français pour Jeanne d'Arc? Tout 
simplement, Shakespeare aurait repris l'assertion de Hall : «she was more to be marveiled at, as a false prophetisse, and seducer of the people then to be honored or worshipped as a sainct sent from God into the realm of Fraunce». Et le même auteur de citer l'historien Paul Émile qui raconte que les citoyens d'Orléans avaient édifié en son honneur «une image ou une idole». Il est de fait qu'alors que l'historiographie officielle française, toute favorable qu'elle soit à la Pucelle, a beau la comparer à telle ou telle héroïne de l'Antiquité païenne ou de l'Ancien Testament, elle a beau mettre en avant toutes ses qualités - piété, chasteté, douceur, sobriété de vie - , elle ne va pas jusqu'à la réputer sainte et évite même d'en parler comme d'une prophétesse: il y a chez cette histographie, depuis le $\mathrm{XV}^{\mathrm{e}}$ siècle, un parti pris de discrétion, de modération qui reflète à l'évidence le point de vue de la monarchie des Valois (et de l'Église de France) sur cet épisode certes honorable mais qu'il convient de contenir à l'intérieur de certaines limites. C'est au contraire l'historiographique de tradition bourguignonne qui insiste lourdement sur l'incroyable crédulité des Français, s'imaginant qu'avec elle Dieu lui-même était descendu sur terre ${ }^{5}$. Dans la même veine, un peu plus loin dans la pièce, Shakespeare place dans la bouche de Charles les paroles suivantes:

We'll set thy statue in some holy place

And have thee reverenc'd like a blessed saint (III.iii).

Après ces sublimes paroles, prêtées, non sans malice, au dauphin, les rapports entre Charles et la Pucelle ne tardent pas à prendre un tour franchement comique: le dâuphin commence par proposer à Jeanne de partager avec lui son royaume, puis son lit, en sorte que, lors de la reprise d'Orléans par les Anglais, il est fait allusion à la fuite précipitée du couple, surpris en chemise comme deux amoureux ordinaires.

Dans tout son comportement. la Pucelle montre de la 
hardiesse, de la détermination, mais aussi un singulier penchant pour la séduction et la tromperie: les attributs du Diable. Il y a tout un passage où, avec des accents dignes du Quadrilogue invectif d'Alain Chartier, elle entreprend de convaincre le duc de Bourgogne de rompre avec l'alliance anglaise et de se réconcilier avec le dauphin en retournant à sa vraie nature de Française:

Look on thy country, look on fertile France, And see the cities and the towns defaced By wasting ruin of the cruel foe.

As looks the mother on her lowly babe When death doth close his tender dying eyes, See, see the pining malady of France;

Behold the wounds, the most unnatural wounds, Which thou thyself hast given her woful breast.

$O$, turn thy edged sword another way.

Strike those that hurt, and hurt not those that help.

One drop of blood drawn from thy country's bosom Should grieve thee more than streams of foreign Return thee therefore with a flood of tears, gore: And wash away thy country's stained spots (III.iii).

Tout le passage est superbe, et la Pucelle sait admirablement jouer sur trois registres: la France comme une mère désolée, le fait que le Bourguignon est fondamentalement un Français, le fait que la victoire anglaise ne lui rapportera rien. Mais on s'aperçoit que ce faisant, elle a été la grande tentatrice qui a amené la Bourgogne à rompre un serment au roi d'Angleterre qu'il venait à nouveau de prêter, à l'instigation de Talbot, tandis que les formules ne manquent pas montrant le cynisme de son discours et de son attitude ainsi que sa capacité à enchanter, au sens propre du terme, son interlocuteur: «By fair persuasions mix'd with sugar's words / We will entice the duke of Burgundy / To leave the Talbot and to follow us » 
(Pucelle); «Speak, Pucelle, and enchant him with thy words» (Charles); «Either she hath bewitch's me with her words, / Or nature makes me suddenly relent» (Bourgogne); «Done like a Frenchman: turn, and turn again» (Pucelle).

Dans le camp anglais, le jeune Talbot, à l'égard de Jeanne d'Arc, prend l'attitude la plus sage: refuser le combat, se dérober à ses défis. Ainsi s'exprime la Pucelle elle-même:

One I encounter's him, and thus I said:

«Thou maiden youth, be vanquish'd by a maid.»

But with a proud majestical high scorn, He answered thus: «Young Talbot was not born To be the pillage of a giglot wench.» So, rushing in the bowels of the French, He left me proudly, as unworthy fight (IV.vii).

Tout ce passe comme si Jeanne d'Arc était invincible tant qu'elle était protégée par ses démons. Mais, une fois Talbot mort, alors que tout sourit en apparence aux Français, déjà prêts à marcher sur Paris, c'est en vain que la Pucelle fait appel aux forces du mal. Celles-ci, accourues, demeurent silencieuses. La scène où Jeanne leur offre successivement un de ses membres, son corps tout entier, enfin son âme, est là pour révéler sa vraie nature, sur laquelle il était encore possible, à la limite, de s'interroger: depuis longtemps déjà elle a bien partie liée avec les démons

This speedy and quick appearance argues proof

Of your accustom'd diligence to me.

Now, ye familiar spirits, that are call'd

Out of the powerful regions under earth,

Help me this once, that France may get the field (IV.iii).

Ces démons, qu'elle a nourris de son sang, l'ont cette 
fois abandonnée, et ses vieilles incantations sont désormais impuissantes. En un ultime corps-à-corps, la Pucelle est vaincue par le duc d'York et faite prisonnière.

On voit mal comment interpréter les derniers moments de Jeanne d'Arc tels que Shakespeare les met en scène dans un sens qui lui soit favorable. En effet, elle a recours, pour sauver sa vie, à toute une série de subterfuges, d'ailleurs si grossiers que ses vainqueurs n'ont aucun mal à les déjouer. Voici d'abord qu'un berger se présente: il s'agit naturellement du père de la Pucelle: mais celle-ci refuse farouchement de le reconnaître, s'affirmant en revanche de race royale ${ }^{6}$. Elle proteste encore de la pureté virginale de son sang qui criera vengeance au Ciel si on la condamne à mort. Mais voyant que de tels propos n'ébranlent pas la détermination de ses adversaires, elle change brusquement de tactique: Shakespeare, reprenant une version qui fait son apparition dans l'historiographie anglaise vers les années $1470^{7}$, la montre se prétendant enceinte. Il convient donc de surseoir pendant quelques mois à son exécution. Et le père de cet enfant qu'elle porte n'est, déclare-t-elle, ni le dauphin ni le duc d'Alençon mais le roi René. Un tel procédé achève de la disqualifier, et York peut l'envoyer à la mort sans risque de susciter chez les spectateurs la moindre réaction de sympathie.

C'est dire que, dans sa présentation de Jeanne d'Arc, Shakespeare combine l'image de la Pucelle comme sorcière et l'image de la Pucelle comme fille de joie, ou courtisane. On peut simplement lui savoir gré, par rapport à ses sources, de ne voir en elle ni une fille d'auberge ni une femme cruelle et de ne pas insister sur l'anti-modèle qu'elle pouvait symboliser par rapport, comme le dit Hall, aux trois qualités propres au véritable idéal féminin: «modestie, compassion, comportement féminin». La Jeanne d'Arc de Henry VI n'est ni une comparse ni une mascotte: elle est une virago déguisée en Vénus, elle est l'âme, rusée, diabolique mais aussi séduisante et déterminée, de la France du dauphin Charles.

Avec cette pièce, Shakespeare a composé une œuvre 
vigoureusement nationaliste. En particulier, il est un thème qui n'apparaît pour ainsi dire nulle part: l'idée que la grande querelle franco-anglaise semblait avoir été réglée, apparemment en accord avec l'un et l'autre des protagonistes, par le traité de Troyes, par ce que les sources contemporaines appellent, dans le camp de Henry VI, la «paix finale» de Troyes, en sorte que, dans cette perspective, le dauphin et ses partisans - les Armagnacs - sont fondamentalement des rebelles qui décident de rallumer une guerre en voie de s'éteindre. Pour Shakespeare au contraire, Henry VI se fait couronner à Paris en roi conquérant plus qu'en roi légitime. Son droit sur le royaume de France dérive de sa généalogie et non d'un acte juridique tel que le traité de Troyes.

Au delà des libertés que Shakespeare prend à l'évidence avec l'authentique succession des événements, au delà des fantaisies, de sa chronologie et de sa géographie, il n'en demeure pas moins que sa façon de peindre la guerre trouve plus d'un écho chez le spécialiste de la fin du Moyen Âge. Négligeons les éventuels anachronismes, et remarquons que le discours shakespearien sur la guerre, par les valeurs qu'il exprime, par les comportements qu'il évoque, par les pratiques auxquelles il se réfère, n'est pas fondamentalement différent du discours sur la guerre propre aux chroniqueurs de la fin du Moyen Âge. De cette concordance dans le ton, dans l'accent, plusieurs raisons peuvent rendre compte: ou bien il existe une certaine ressemblance, dans la réalité, entre la guerre sous les Lancastres et la guerre sous Elisabeth I; ou bien d'une manière générale, encore à la fin du XVI ${ }^{e}$ siècle, le discours sur la guerre, quels qu'en soient les auteurs, est proche de celui en vigueur un siècle ou deux plus tôt; ou bien encore il revient au génie de Shakespeare d'avoir rassemblé ici le legs tout entier du Moyen Âge finissant dans le domaine de la guerre comme il l'a rassemblé ailleurs dans celui de l'amour du sacré ou de l'imaginaire. 


\section{NOTES}

(1) Voir en dernier lieu Ph. Contamine, La guerre de Cent ans, 5e éd., Paris, 1989, et C.T. Allmand, The Hundred years War. England and France at War, c. 1300-c. 1450, Cambrigde, 1988.

(2) Expression préférable à celle de «guerre des deux Roses», habituellè en France.

(3) Ph. Contamine, «Naissance d'une historiographie. Le souvenir de Jeanne d'Arc en France et hors de France, depuis le procès de son innocence (1455-1456) jusqu'au début du XVI ${ }^{c}$ siècle», Francia, 15 (1987).

(4) Au XVI ${ }^{\mathrm{e}}$ siècle, c'est l'image de Jeanne d'Arc chef de guerre, héroïne, qui l'emporte. «Le temps des panaches», dit Jacqueline Melet-Sanson dans sa contribution au catalogue de l'exposition Images de Jeanne d'Arc. Hommage pour le $550^{\circ}$ anniversaire de la libération d'Orléans et du Sacre, Paris, Hôtel de la Monnaie, juillet-septembre 1979.

(5) Ph. Contamine, art. cité.

(6) Pure imagination de Shakespeare, reprise, comme on sait, mais beaucoup plus tard, par toute une littérature de fiction, aujourd'hui proliférante.

(7) $\mathrm{Ph}$. Contamine, art. cité. 